Available online at GSC Online Press Directory

GSC Biological and Pharmaceutical Sciences

e-ISSN: 2581-3250, CODEN (USA): GBPSC2

Journal homepage: https://www.gsconlinepress.com/journals/gscbps

(REVIEW ARTICLE)

\title{
Can vitamin B12 be an adjuvant to COVID-19 treatment?
}

\author{
Lopes Monyck Jeane dos Santos ${ }^{1, *}$ \\ Museu Paraense Emílio Goeldi. Av. Magalhães Barata, 376, 66040-170, São Braz, Belém, Pará, Brazil.
}

Publication history: Received on 27 May 2020; revised on 10 June 2020; accepted on 11 June 2020

Article DOI: https://doi.org/10.30574/gscbps.2020.11.3.0155

\begin{abstract}
COVID-19 has become an international pandemic and is causing a worldwide public health emergency; therefore, an effective treatment is urgently needed. The hypothesis is that Covid virus interferes with the cobalamin metabolism, causing symptoms of cobalamin deficiency. This is plausible to infer, because there are symptoms of vitamin B12 deficiency that are similar to those of COVID-19. These symptoms include increase oxidative stress, homocysteine concentration, activation of the coagulation cascade, thrombocytopenia, elevated lactate dehydrogenase (LDH), low reticulocyte count, intravascular coagulation thrombosis, vasoconstriction, renal and pulmonary vasculopathies, which can result in respiratory, gastrointestinal and central nervous system disorders. Research shows that high doses of methylcobalamin is the treatment for symptoms of vitamin B12 deficiency. Thus, an additional hypothesis is that treatment with vitamin B12, especially methylcobalamin, would reduce Covid's damage to infected patients. Hence, in this review article it is suggested that methylcobalamin (vitamin B12) may serve as an attenuator to COVID-19 symptoms. Clinical studies are required to confirm this hypothesis.
\end{abstract}

Keywords: Coronavirus disease 2019; Vitamin; Methylcobalamin; Methyl-12; Cobalamin

\section{Introduction}

COVID-19 is highly transmissible in humans; mostly presented with mild flu-like symptoms, but some patients, especially the elderly and people with underlying diseases, evolve critical condition and rapidly develop acute respiratory distress syndrome, respiratory failure, multiple organ disorder and death $[1,2]$. Globally, there are more than 7.000.000 confirmed cases of Coronavirus disease-2019 (COVID-19 / SARS-CoV-2), with more than 400.000 deaths, in 188 countries/regions [Coronavirus Johns Hopkins, https://coronavirus.jhu.edu/, Last accessed on 11/06/2020]. Due to their effects on impairing viral replication, enhancing anti-inflammatory immune responses and reducing pro-inflammatory responses [3,4], vitamins are expected to increase immunity against COVID-19. Vitamin B12 (cobalamin) is an immunomodulator for cellular immunity, acting as a support to hematopoietic, nervous and immune systems $[4,5]$. Highest cobalamin deficit may occur in the elderly, who have difficulty in absorbing this vitamin from food, due to the lack of gastric acid or the intrinsic factor needed to active absorption of B12 [5-8]. This condition could be a likely explanation to the high vulnerability of the elderly to COVID-19 infection [1,2].

Kandeel and Al-Nazawi [9] used the molecular modeling tools and reported that ribavirin, telbivudine, vitamin B12 and nicotinamide can be a potential treatment for COVID-19. Narayanan and Nair [10] suggest that vitamin B12 (methylcobalamin) may be a possible inhibitor of the RNA-dependent-RNA polymerase activity of the SCV2-nsp12 enzyme, and that the inhibition of this enzyme can result in lower viral infection and reduce the severity of the COVID19 disease. Zhang et al. [11] suggest that melatonin, an anti-inflammatory and anti-oxidative molecule, has the potential to treat COVID-19 patients. Research proves the increase in melatonin synthesis with the use of vitamin B12 [12,13].

\footnotetext{
* Corresponding author: Lopes Monyck Jeane dos Santos; phone: +55 91982113700; e-mail: monycklopes@museu-goeldi.br
} 
I had severe vitamin B12 deficiency symptoms. After a long and tiring search, I started vitamin B12 therapy, with cyanocobalamin injections, until I started to use sublingual methylcobalamin, with excellent clinical results. Since this treatment, as I am a scientist and researcher in the biological field, I started to research scientific articles on vitamin B12. During this pandemic, I observed similarities between COVID-19 responses and vitamin B12 deficiency symptoms. So, I started to review the literature on how this virus could interfere with vitamin B12 metabolism, and consequently, cause damage to the human body. Thus, emerged the hypothesis that Covid virus interferes in the cobalamin metabolism, causing symptoms of cobalamin deficiency. An additional hypothesis is that treatment with vitamin B12, especially methylcobalamin, could reduce Covid's damage. However, there is currently insufficient evidence regarding the effectiveness of B Vitamins in the treatment of COVID-19. There is no mention of B vitamins in the WHO Interim Guidance, US CDC Clinical Interim Guidelines and Chinese Clinical Guidance for COVID-19 management [14]. The lack of targeted treatment for COVID-19 requires assessing all potential modalities to reduce the damage caused by this disease. In this review article I suggests that methylcobalamin form of vitamin B12 may serve as an attenuator of COVID19 symptoms.

\section{Vitamin B12}

Vitamin B12, a crystallisable cobalt-complex called cobalamin, is the largest and most complex of all the vitamins. This vitamin is not used directly in the human body, as it needs to be translated into activating forms such as hydroxo-, adenosyl- and methylcobalamin $[15,16]$. Cobalamin is produced exclusively by microbial synthesis in the digestive tract. Therefore, animal protein products are the natural sources of vitamin B12 in the human diet $[6,16]$. This vitamin is essential for the formation of red blood cells, maintenance a healthy nervous system, in cell division, myelin synthesis and cellular growth and reproduction and the rapid synthesis of DNA $[4,6]$.

Dietary cobalamin is released in the acidic environment of the stomach where it is rapidly complexed to the binding protein and transporter haptocorrin, also referred to as the transcobalamin. Cobalamin continues its route in the gastrointestinal track and dissociates from haptocorrin transporter, under the action of pancreatic proteases, followed by its association in the intestine with the intrinsic factor, which is essential for ileal absorption of cobalamin $[5,16,17]$.

Cobalamin metabolism is complex and requires many processes and steps, if any one of these are not present, it may lead to cobalamin deficiency $[15,16]$. The inability to release cobalamin from food, or its binding proteins, is described as the syndrome of food-cobalamin malabsorption [16]. There are many factors that can interfere on cobalamin absorption, such as atrophic gastritis (autoimmune gastritis), Helicobacter pylori, intestinal microbial proliferation [16,17], celiac disease, inflammatory bowel disease, alcoholism and prolonged pharmacological treatments [5]. There are also specific polymorphisms or genetic mutations that can interfere on cobalamin absorption [17]. In addition, there are several medications that impair cobalamin absorption and effectiveness, such as antibiotics, colchicines, nonsteroidal anti-inflammatory drug (NSAIDs), nitrates, fluorouracil, barbiturates, pyrimethamine, aminoglycosides, proton pump inhibitors, antihyperglycemic medications (metformin) and anticonvulsants $[5,13]$.

Low cobalamin levels will increase methylmalonic acid and homocysteine [17], increasing the producing reactive oxygen species. These byproducts lead to endothelial dysfunction, platelet activation and increased tissue factor expression with subsequent activation of the coagulation cascade [8]. Some cases of deficiency may lead to megaloblastic anemia, disruption of neuron myelin sheath integrity, reduced protective immune response, neurological problems and degeneration of the spinal conditions $[4,7,17]$. There are also subclinical cobalamin deficiency signs such as psychiatric symptoms, depression, psychosis and occasionally suicidal thoughts [17]. Other symptoms are signs of mechanical hemolysis, thrombocytopenia, elevated lactate dehydrogenase (LDH), low reticulocyte count, intravascular coagulation thrombosis, vasoconstriction and renal and pulmonary vasculopathies $[8,18]$.

The natural course of vitamin B12 deficiency is not clearly understood [17]. Level of vitamin B12, holotranscobalamin, methylmalonic acid and homocysteine can help diagnose cobalamin deficiency even with normal levels of vitamin B12 in the blood $[8,19]$. In addition, cobalamin deficiency can be diagnosed by the Schilling test, used for the diagnoses of the syndrome of food-cobalamin malabsorption [16]. Holotranscobalamin (referred to as active vitamin B12) is preferred for the detection of subclinical B12 deficiency [16,19]. However, normal levels of serum B12 and methylmalonic acid do not exclude symptomatic B12 deficiency $[5,17,20,21]$. That is why, it is still difficult to establish a correct diagnosis of vitamin B12 deficiency and prescribe the right treatment $[5,8,17]$. So, unfortunately, so many cases of B12 deficiency are overlooked or, sometimes, even misdiagnosed [17]. 


\section{Pathogenesis of COVID-19 and the rationale for Vitamin B12 treatment}

The clinical symptoms of COVID-19 include fever, respiratory distress syndrome, cough, fatigue and gastrointestinal infection symptoms [1,2]. Clinical laboratory findings include impaired liver function, lymphopenia, arrhythmia, shock, acute kidney injury, acute cardiac injury, liver dysfunction and secondary infection, increased PCR, LDH, D-dimer and ferritin levels; and reduction in platelet counts and erythrocyte sedimentation rate [2,4,22]. Covid-19 disease induced cytokine storm triggers the excessive production of pro-inflammatory cytokines, cause precipitates neutrophil and macrophage sequestration, pulmonary hyaline membrane formation and diffuse alveolar wall thickening, increase iron entry into the lungs and multiple organ dysfunction [2,4,22,23]. COVID-19 also cause pneumonia, while also causing acute myocardial injury, chronic damage to the cardiovascular system and myocardium [23].

It is reasonable to suppose that Covid virus interferes with cobalamin metabolism, possibly impairing the intestinal microbial proliferation, causing symptoms of cobalamin deficiency. This is plausible to infer, because there are symptoms of vitamin B12 deficiency that are similar to those of COVID-19 infection, such as the increase of oxidative stress, homocysteine concentration, activation of the coagulation cascade, thrombocytopenia, elevated lactate dehydrogenase (LDH), low reticulocyte count, intravascular coagulation thrombosis, vasoconstriction and renal and pulmonary vasculopathies $[8,18]$. These which can result in respiratory, gastrointestinal, and central nervous system disorders [17]. Research shows that high dose of methylcobalamin is the treatment for symptoms of B12 deficiency $[5,7,16,17]$. Based on research reports, it could be inferred that treatment with Vitamin B12, especially methylcobalamin, could reduce Covid's damage.

\section{Treatment}

Vitamin B12 therapy reduces oxidative stress, inflammation and microvascular disease associated with hyperhomocysteinemia [21]. However, this vitamin metabolism is complex and requires many processes and steps, any one of which, if not present, may lead to cobalamin deficiency. Cyanocobalamin is the form of vitamin B12 present in many supplements, in the liver it is converted to methylcobalamin, replacing cyanide with methyl group; but, sometimes the liver cannot convert cyanocobalamin into adequate amount of methylcobalamin needed by the human body [13,21].

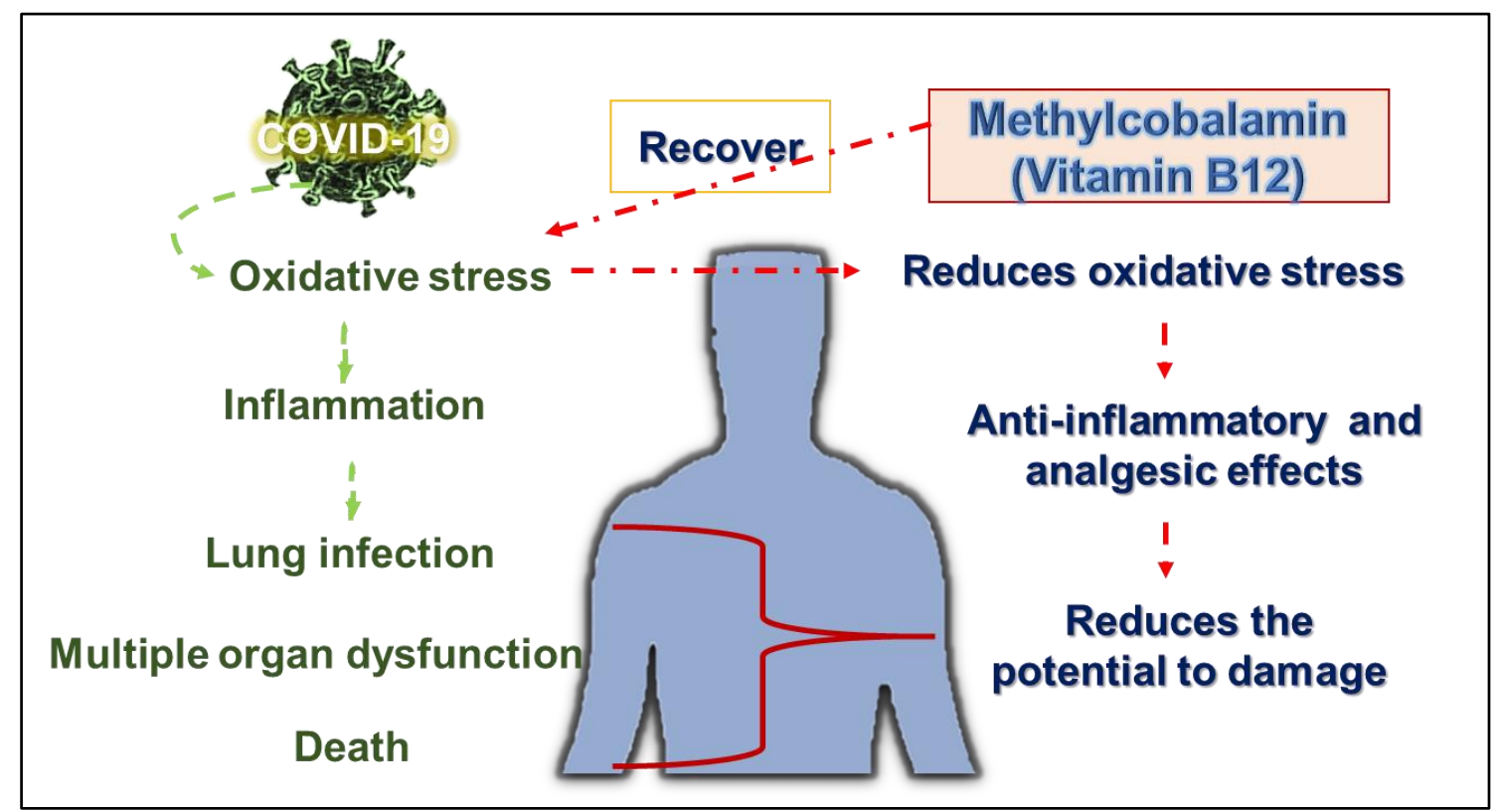

Figure 1 Summary of vitamin B12 (methylcobalamin) role in reducing Covid's damage.

Methylcobalamine forms adenosylcobalamine, the other form of vitamin B12 for mitochondrial energy production [13]. Its methyl group stimulates serotonin creation, a neurotransmitter which is responsible for mood enhancement and protecting the brain from damage by excitotoxins [13]. It also acts in the integrity of myelin, neuronal function, proper red blood cell formation and DNA synthesis [13,15]. Methylcobalamin may be considered as a supplement, with potential benefits in the treatment of vitamin B12 deficiency, Alzheimer's disease syndromes, memory improvement, neuronal protection, nerve regeneration, visual disfunction, rheumatoid arthritis, Bell's palsy and sleep-wake rhythm disorder $[15,24]$. 
Methylcobalamin is the active form of vitamin B12. It is more effective than other analogs; converts homocysteine to methionine and S-adenosylmethionin and reduces oxidative stress [13,15,21]. Thus, methylcobalamin treatment would probably enable a faster recovery in COVID-19 infected patients. This treatment would reduce oxidative stress, act as an anti-inflammatory and analgesic agent [24] and could minimize the potential for damage caused by this disease, such as lung infection, multiple organ dysfunction and death (Figure 1).

The pharmacological treatment of vitamin B12 deficiency occurs with high doses (or 1000-2000 mcg/day) for at least 3 months, and can be administered orally, parenterally, intranasal and sublingual $[5,7,13,16,17,20,21]$. Oral supplementation may increase the serum vitamin B12 level but often not enough to replenish the vitamin B12 levels in the tissues $[17,20]$. On the other hand, sublingual absorption is most efficient, because it bypasses the initial passes of the hepatic metabolic process, providing more bioavailability and rapid onset of action, increasing blood levels in 10 to 15 minutes, acting much faster than when ingested orally [25]. Cobalamin is a water-soluble vitamin, so the excess absorbed is then excreted with urine, there is no known health risk from consuming very high intakes $[5,7]$.

\section{Conclusion}

Vitamin B12 therapy reduces oxidative stress, improves circulation and acts as an anti-inflammatory and analgesic, thus probably reducing the damage of COVID-19 patients. Hence, vitamin B12 (Methylcobalamin) has a high safety profile; its use by COVID-19 patients would possibly be highly beneficial. Considering the hypothesis that COVID is interfering in vitamin B12 absorption, soon those infected with this virus will develop symptoms that are also common in known cases the deficiency of this vitamin. Therefore, it is possible to infer that research on the use of methylcobalamin in patients with COVID-19 infection could become an alternative treatment to this pandemic. Clinical studies are required to confirm this hypothesis.

\section{Compliance with ethical standards}

\section{Acknowledgments}

I thank Moacyr Bernardino Dias-Filho for support. I also thank MCTIC/CNPq (301341/2020-6).

\section{Disclosure of conflict of interest}

The Author declare no conflict of interest.

\section{References}

[1] Guo YR, Cao QD, Hong ZS, Tan YY, Chen SD, Jin HJ, Tan KS, Wang DY and Yan Y. (2020). The origin, transmission and clinical therapies on coronavirus disease 2019 (COVID-19) outbreak - an update on the status. Military Medical Research, 7(11), 1-10.

[2] Mehta P, McAuley DF, Brown M, Sanchez E, Tattersall RS and Manson JJ. (2020). COVID-19: consider cytokine storm syndromes and immunosuppression. International Health Regulations, 395, 1033-1034.

[3] Carella AM, Benvenuto A, Lagattolla V, Marinelli T, De Luca P, Ciavarrella G, Modola G, Di Pumpo M, Ponziano E and Benvenuto M. (2020). Vitamin supplements in the Era of SARS-Cov2 pandemic. GSC Biological and Pharmaceutical Sciences, 11 (2), 07-19.

[4] Stipp MM. (2020). SARS-CoV-2: Micronutrient Optimization in Supporting Host Immunocompetence. International Journal of Clinical Case Reports and Reviews, 2 (2), 1-11.

[5] Rizzo G and Laganà AS. (2020). A review of vitamin B12. Molecular Nutrition, 105-129.

[6] Hanna S, Leonard L and Rajarethinam RP. (2009). Prim Care Companion. J Clin Psychiatry, 11(5), 269-270.

[7] Allen LH. (2018). Efficacy and Safety of Vitamin B12 Fortification. Food Fortification in a Globalized World, 255261.

[8] Sabry W, Elemary M, Burnouf T, Seghatchian J and Goubran H. (2020). Vitamin B12 deficiency and metabolismmediated thrombotic microangiopathy (MM-TMA), Transfusion and Apheresis Science, 59 (102717), 1-4.

[9] Kandeel M and Al-Nazawia M. (2020). Virtual screening and repurposing of FDA approved drugs against COVID19 main protease. Life Sciences, 251 (117627), 1-5. 
[10] Narayanan N and Nair DT. (2020). Vitamin B12 may inhibit RNA-dependent-RNA polymerase activity of nsp12 from the SARS-CoV-2 Virus. Preprints, 1-19.

[11] Zhang R, Wang X, Ni L, Di X, Ma B, Niu S, Liu C and Reiter RJ. (2020). COVID-19: Melatonin as a potential adjuvant treatment. Life Sciences, 250(117583), 1-6.

[12] Yanagihara M, Nakamura M, Usui A, Nishida S, Ito E, Okawa M and Inoue Y. (2014). The Melatonin Receptor Agonist Is Effective for Free-Running Type Circadian Rhythm Sleep Disorder: Case Report on Two Sighted Patients Tohoku. J. Exp. Med, 234, 123-128.

[13] Gupta JK and Sana QS. (2015). Potential Benefits of Methylcobalamin: A Review. Austin Journal of Pharmacology and Therapeutics, 3, 1-4.

[14] Villarruz-Sulit MV and Cabaluna IT. (2020). Should B Vitamins be used in the treatment of COVID-19? Asia pacific center for evidence-based healthcare, 1-2.

[15] Zhang M, Han W, Hu S and Xu H. (2013). Methylcobalamin: A Potential Vitamin of Pain Killer Neural Plasticity, 424651, 1-6.

[16] Andres E and Dali-Youcef N. (2020). Cobalamin (vitamin B12) malabsorption. Molecular Nutrition, 367-386.

[17] Wolffenbuttel BHR, Wouters HJCM, Heiner-Fokkema MR and Klauw MM. (2020). The many faces of cobalamin (vitamin b12) deficiency. Mayo clinic proceedings: innovations, quality \& outcomes, 3(2), 200-214.

[18] Grangé S, Bekri S, Artaud-Macari E, Francois A, Girault C, Poitou AL, Benhamou Y, Vianey-Saban C, Benoist JF, Châtelet V, Tamion F and Guerrot D. (2015). Adult-onset renal thrombotic microangiopathy and pulmonary arterial hypertension in cobalamin C deficiency. The Lancet, 386(9997), 1011-1012.

[19] Campos AJ, Risch L, Nydegger U, Wiesner J, Dyck MVV, Renz H, Stanga Z and Risch M. (2020). Diagnostic accuracy of holotranscobalamin, vitamin b12, methylmalonic acid, and homocysteine in detecting b12 deficiency in a large, mixed patient population. Disease Markers, ID 7468506, 1-11.

[20] Naseri M, Sarvari GR, Esmaeeli M, Azarfar A, Rasouli Z and Moeenolroayaa G. (2016). High doses of oral folate and sublingual vitamin B12 in dialysis patients with hyperhomocysteinemia. Journal of Renal Injury Prevention, 5(3), 134-139.

[21] Lopes SC, Gadelha DD, Carvalho MD, Fernandes VO and Montenegro Junior RM. (2019). Vitamin B12 deficiency: metabolic effects, clinical evaluation, and treatment Revista de Medicina da UFC, 59(2), 40-49.

[22] Yilmaz N and Esin E. (2020). Covid-19 and Iron Gate: The Role of Transferrin and Transferrin Receptor.

[23] Zheng Y, Ma Y, Zhang J and Xie X. (2020). COVID-19 and the cardiovascular system. Nature Reviews Cardiology $17,259-260$.

[24] Rahman M, Ferdoush KA, Jahan K, Masood S, Mollika R, Islam S and Orthi SU. (2020). Analgesic effects of vitamin B12: a recent update. Journal of Global Biosciences, 9(4), 7160-7170.

[25] Nibha KP and Pancholi SS. (2012). An Overview on: Sublingual Route for Systemic Drug Delivery International. Journal of Research in Pharmaceutical and Biomedical Sciences, 3(2), 913-923.

\section{How to cite this article}

Lopes MJS. (2020). Can vitamin B12 be an adjuvant to COVID-19 treatment? GSC Biological and Pharmaceutical Sciences, 11(3), 01-05. 